\title{
Study of the Incorporation Behavior of L-Leu and D-Leu in L-Val Crystallization
}

\author{
Jun Sen1,2*, Norimoto Kokubun², Toshimichi Kamei², Kazushige Ohmori², \\ Mitsuhiro Kishino' ${ }^{2}$, Tatsuki Kashiwagi ${ }^{3}$, Masaaki Yokota1, Norihito Doki ${ }^{1}$ \\ ${ }^{1}$ Department of Chemistry and Bioengineering, Iwate University, Morioka, Japan \\ ${ }^{2}$ Research Institute for Bioscience Products \& Fine Chemicals, Ajinomoto Co., Inc., Kawasaki, Japan \\ ${ }^{3}$ Research Institute for Innovation, Ajinomoto Co., Inc., Kawasaki, Japan

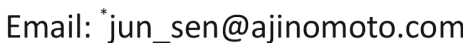

Received 30 May 2016; accepted 10 July 2016; published 13 July 2016

Copyright (C) 2016 by authors and Scientific Research Publishing Inc.

This work is licensed under the Creative Commons Attribution International License (CC BY).

http://creativecommons.org/licenses/by/4.0/

(c) (i) Open Access

\begin{abstract}
Branched-Chain Amino Acids (BCAAs) are essential amino acids mainly produced through fermentation. With respect to BCAA crystallization, the incorporation behavior of L-form BCAAs as a guest amino acid in the crystallization of L-form BCAAs is well known. However, the incorporation behavior of $D$-form BCAAs as a guest amino acid in the crystallization of L-form BCAAs is not clear. In this study, we focused on the crystal conformation and incorporation behavior of D-Leucine (D-Leu) and L-leucine (L-Leu) in the crystallization of L-valine (L-Val). Moreover, the difference in the incorporation behavior was investigated on the basis of the distribution ratio of crystals to mother liquor, and the crystal structure and interaction energy were calculated using a molecular dynamics method. The crystal composed of L-Leu in L-Val formed as a solid solution and L-Leu was substituted into the crystal lattice of L-Val. In the case where D-Leu was added as a guest amino acid during L-Val crystallization, D-Leu was not incorporated into the L-Vallattice because the interaction energy between a D-Leu molecule and the L-Val crystal lattice was substantially greater than that between an L-Leu molecule and the L-Val crystal lattice.
\end{abstract}

\section{Keywords}

Branched Chain Amino Acid, Crystallization, L-Valine, Leucine, Solid Solution

\section{Introduction}

Amino acids are the fundamental building blocks of peptides and proteins and are used innumerous applications

\footnotetext{
*Corresponding author.
}

How to cite this paper: Sen, J., Kokubun, N., Kamei, T., Ohmori, K., Kishino, M., Kashiwagi, T., Yokota, M. and Doki, N. (2016) Study of the Incorporation Behavior of L-Leu and D-Leu in L-Val Crystallization. Advances in Chemical Engineering and Science, 6, 262-268. http://dx.doi.org/10.4236/aces.2016.63026 
including pharmaceuticals, medical food, and fine chemicals. Collectively, L-leucine (L-Leu), L-isoleucine (L-Ile), and L-valine (L-Val) are known as Branched-Chain Amino Acids (BCAAs). BCAAs are essential amino acids and necessary for proper muscle function. Recently, BCAAs have been reported to prevent locomotive syndrome in the elderly [1] [2]. In most cases, BCAAs are commercially produced through fermentation. Small quantities of other amino acids, including other BCAAs, are also produced as impurities during the BCAA fermentation process. The target BCAAs must be separated from impurity-containing mixtures to be shipped as a product. In BCAA crystallization, other BCAAs are known to be difficult to separate because of their common components and tendency to form solid solutions [3]. In this case, "solid solution" refers to a crystal structure produced by random inclusion of impurities into the lattice of the host amino acid (i.e., the target amino acid) crystal. The formation of a solid solution during BCAA crystallization has been reported to be a consequence of the host and the guest amino acids (impurity amino acids) having similar branched hydrophobic side chains and crystal structures [4]-[8]. Co-crystals with a 1:1 ratio of D-form and L-form BCAAs have been reported [9]-[11]; however, quantitative evaluations of their mutual incorporation behavior are rare.

In this report, we focus on the crystal conformation and incorporation behavior of D-leucine (D-Leu) in crystalline L-Val (D-Leu/L-Val). For comparison, the incorporation behavior of L-Leu in L-Val crystals was also studied.

\section{Experimental}

\subsection{Materials}

The D-Leu used in this study was a special grade (purity $>98.0 \%$ ) and purchased from WAKO Pure Chemicals. L-Leu and L-Val used in this study were pharmaceutical grade and purchased from AJINOMOTO Co., Inc.

\subsection{Methods}

The experimental flow is shown in Figure 1. First, a $30 \mathrm{~g} / \mathrm{L} \mathrm{L-Val} \mathrm{aqueous} \mathrm{solution} \mathrm{was} \mathrm{prepared.} \mathrm{L-Leu} \mathrm{or}$ D-Leu was added to this solution and the resulting solution was stirred. The solution was then evaporated until the concentration of $\mathrm{L}-\mathrm{Val}$ in the solution $\left(C_{\mathrm{L}-\mathrm{Val}}\right)$ was $20 \mathrm{wt} \%$. Crystals formed in the solution. The resulting slurry was subsequently placed in a $50^{\circ} \mathrm{C}$ water bath, cooled from $50^{\circ} \mathrm{C}$ to $10^{\circ} \mathrm{C}$ at a cooling rate of $10^{\circ} \mathrm{C} / \mathrm{h}$, and

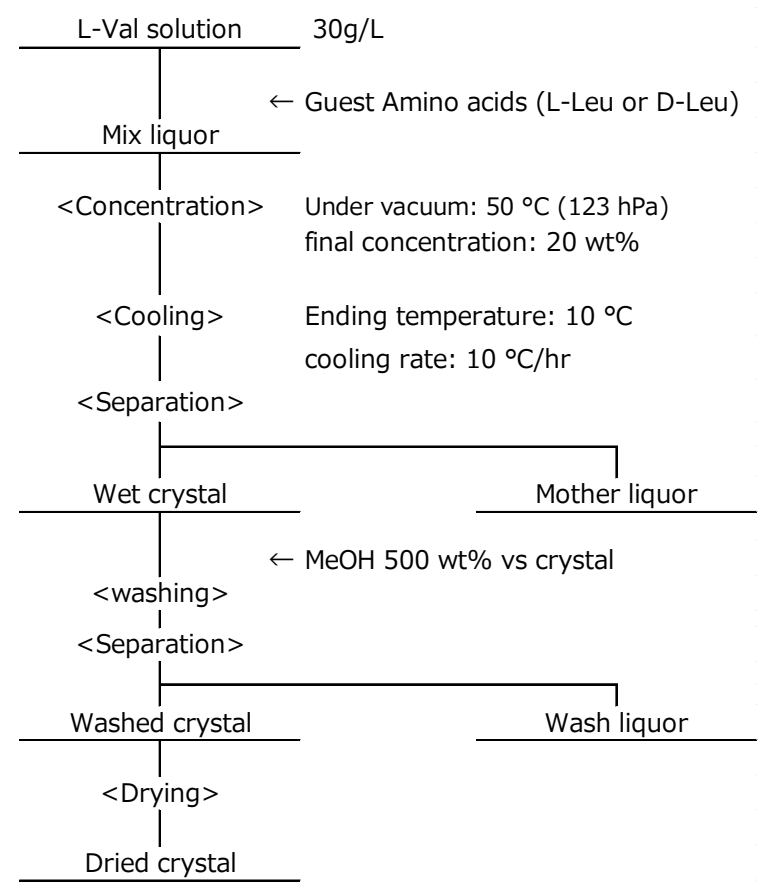

Figure 1. Experimental flow of L-Leu or D-Leu crystallization in L-Val. 
then maintained at $10^{\circ} \mathrm{C}$ overnight. The slurry was filtrated using $0.45 \mu \mathrm{m}$ membrane filters. The wet crystals were washed using $\mathrm{MeOH}$. The concentrations of L-Leu, D-Leu, and L-Val were analyzed by high-performance liquid chromatography (HPLC; column: MCIGEL CRS10W; column temperature: $30^{\circ} \mathrm{C}$; detection wavelength: $254 \mathrm{~nm}$; measuring time: $15 \mathrm{~min}$; mobile phase: $1 \mathrm{mM} \mathrm{CuSO}_{4}$ aq./MeOH [v/v] = 85/15; flow rate: $1 \mathrm{~mL} / \mathrm{min}$ ). The crystal structure was analyzed using powder X-ray diffraction (PXRD: Bruker D2 PHASER, CuK $\alpha, 30 \mathrm{kV}$ ) and the crystal shape was analyzed by scanning electron microscopy (SEM; JEOL JSM-6510).

\section{Results and Discussion}

\subsection{Solid-Liquid Equilibria of L-Leu/L-Val and D-Leu/L-Val}

The initial experimental system is represented as the molar ratio between the guest amino acid and the host amino acid. For example, L-Leu crystallization in L-Val is represented as "L-Leu/L-Val". A comparison of the incorporation behaviors of L-Leu/L-Val and D-Leu/L-Valis shown in Figure 2, where the ratios of guest amino acids to L-Val in the crystals are plotted against those in the mother liquor at equilibrium. In the case of L-Leu used as aguest amino acid (blue solid circles), L-Leu was incorporated into L-Val crystals. The impurity content in the crystals was linearly plotted against tha tin the mother liquor. By contrast, in the case where D-Leu was used as aguest amino acid (red open circles), all D-Leu remained in the mother liquor. When the molar ratio of D-Leu to L-Val (represented as $x_{\text {D-Leu }}$ ) in the mother liquor was less than 0.12 , D-Leu was not incorporated into L-Val crystals. However, when $x_{\text {D-Leu }}$ was greater than 0.12 , D-Leu crystallized with L-Val crystals. Thus, the solubility of D-Leu in the mother liquor was $x_{\mathrm{D} \text {-Leu }}=0.12$.

In summary, L-Leu was incorporated in a certain ratio of $x_{\text {L-Leu }}$ in the mother liquor. D-Leu was not incorporated when its concentration in the mother liquor was less than the solubility limit; however, D-Leu was crystallized when its concentration in the mother liquor was greater than the solubility limit. In this report, we mainly discuss cases where $x_{\mathrm{L}-L e u}<0.12$ and $x_{\mathrm{D} \text {-Leu }}<0.12$ in the mother liquor.

\subsection{Crystal Conformation}

The structure of crystals obtained by crystallization was analyzed by PXRD (Figure 3 ). The molar ratio between $\mathrm{L}-\mathrm{Val}$ and L-Leu in the crystal is denoted as L-Leu/L-Val in this figure. The diffraction angles $(2 \theta)$ of the (001) and (002) peaks in the pattern of L-Val were detected at $2 \theta=7.3^{\circ}$ and $14.6^{\circ}$, respectively. The diffraction angles of the (001) and (002) peaks in the pattern of L-Leu were detected as $2 \theta=6.0^{\circ}$ and $12.1^{\circ}$, respectively. In the case of L-Leu incorporated into L-Val crystals, these peaks were shifted to lower angles than those of singlecrystalline L-Val and the extent of their shift was dependent on $x_{\mathrm{L}-\mathrm{Leu}}$ in the crystals. The relationship between the diffraction angle and lattice spacing, known as Bragg's law, is shown in Equation (1):

$$
2 d \sin \theta=n \lambda
$$

where $d$ is the lattice spacing, $n$ is an integer $(n=1,2,3, \cdots)$ representing the order of diffraction, and $\lambda$ is the X-ray wavelength (CuK $\alpha$ : $1.5418 \AA$ ). As $\theta$ decreases, $d$ increases. Our PXRD results show that L-Leu was

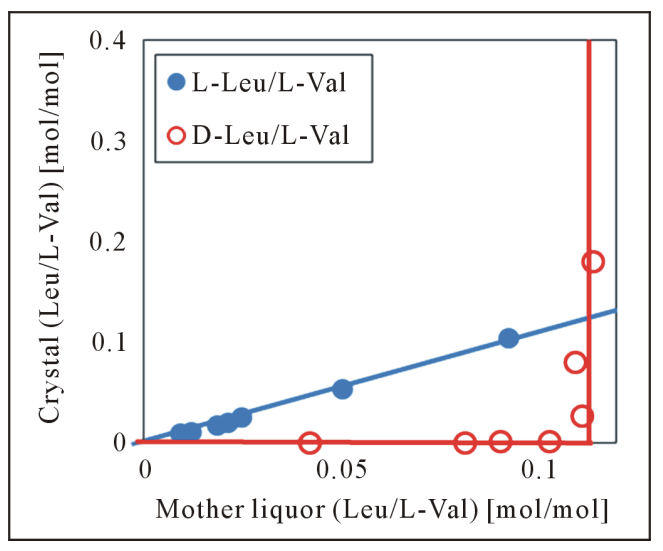

Figure 2. Plot of the distribution ratio of Leu and $\mathrm{L}$-Val in crystals and mother liquors. 


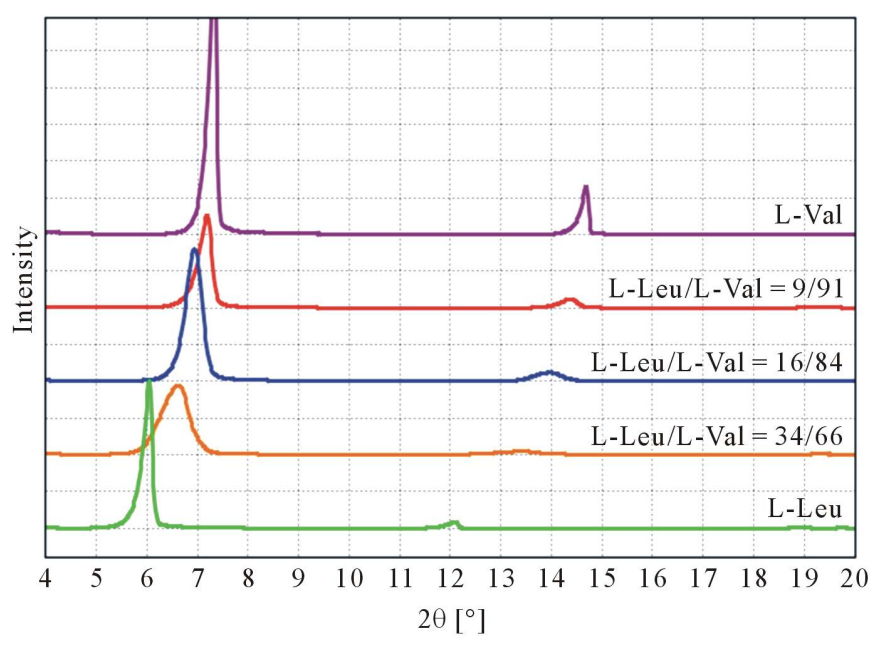

Figure 3. PXRD patterns of L-Leu/L-Val crystals. The molar ratio of L-Val and L-Leu in the crystal is represented as "L-Leu/L-Val" $[\mathrm{mol} / \mathrm{mol}]$ in the figure.

incorporated into the unit cell of an L-Val crystal and that the $c$-axis expanded. Moreover, the incorporation of greater amounts of L-Leu into L-Val crystals resulted in broader peaks. It was speculated that the regularity of the crystal array decreased because L-Leu was randomly incorporated into the L-Val crystal lattice. We therefore considered these crystals to be a solid solution. L-Val and L-Leu peaks were not detected in any of the PXRD patterns of L-Leu/L-Val. Therefore, L-Val and L-Leu single crystalsdid not form; only the L-Leu/L-Val solid solution crystallized.

\subsection{Crystal Morphology}

The crystal shape differed markedly between the L-Leu/L-Val crystal and the D-Leu/L-Val crystal (Figure 4). When $x_{\text {D-Leu }}$ was less than 0.12 in the mother liquor, the molar ratio of the obtained crystal was $\mathrm{D}-\mathrm{Leu} / \mathrm{L}-\mathrm{Val}=$ 0.03/99.97. D-Leu was hardly incorporated into L-Val by crystallization. The crystal clearly exhibited a hexagonal-plate morphology, which was identical to that of the L-Val single crystal. However, vaguely outlined fine crystals were obtained in the case of L-Leu/L-Val crystallization; this morphology was a result of the formation of a solid solution. In the L-Leu/L-Val solid solution, L-Leu was randomly incorporated into the L-Val crystal lattice. Because the side chain of L-Leu is larger than that of L-Val, L-Val crystal growth was stopped at the point of L-Leu incorporation, disturbing the normal growth of L-Val crystals. Therefore, the L-Leu/L-Val solid solution crystallized as vaguely outlined and fine crystals.

\subsection{Estimation of Interaction Energy}

L-Leu was incorporated into L-Val crystals, whereas D-Leu was not. We speculate that this difference in incorporation behavior was due to the magnitude of the interaction energy between a molecule of the guest amino acid (L-Leu or D-Leu) and an L-Val crystal. The interaction energy is correlated with the stability of the crystal structure: when the interaction energy is lower, the crystal structure is more stable.

We calculated the interaction energy on the basis of this assumption. The interaction energy is difficult to determine experimentally; we therefore calculated it using a molecular dynamics package (Materials Studio, from Dassault Systemes Biovia K.K.). The L-Val $4 \times 4 \times 2$ crystal structure was drawn and optimized geometrically using the cvff function, which is a generalized valence potential function used to model small organic crystals and gas-phase structures such as amides, carboxylic acids, proteins, and a wide range of organic compounds [12] [13]. A guest amino acid was used to replace one of the existing L-Val (100) surfaces (structure A). The crystal lattice in which the guest compound was removed from structure A was constructed (structure B). The crystal lattice in which all L-Val except the guest compound was removed from structure A was also constructed (structure C). Structures A-C are shown in Figure 5. The interaction energy $\left(E_{\mathrm{I}}\right)$ was estimated from Equation (2): 

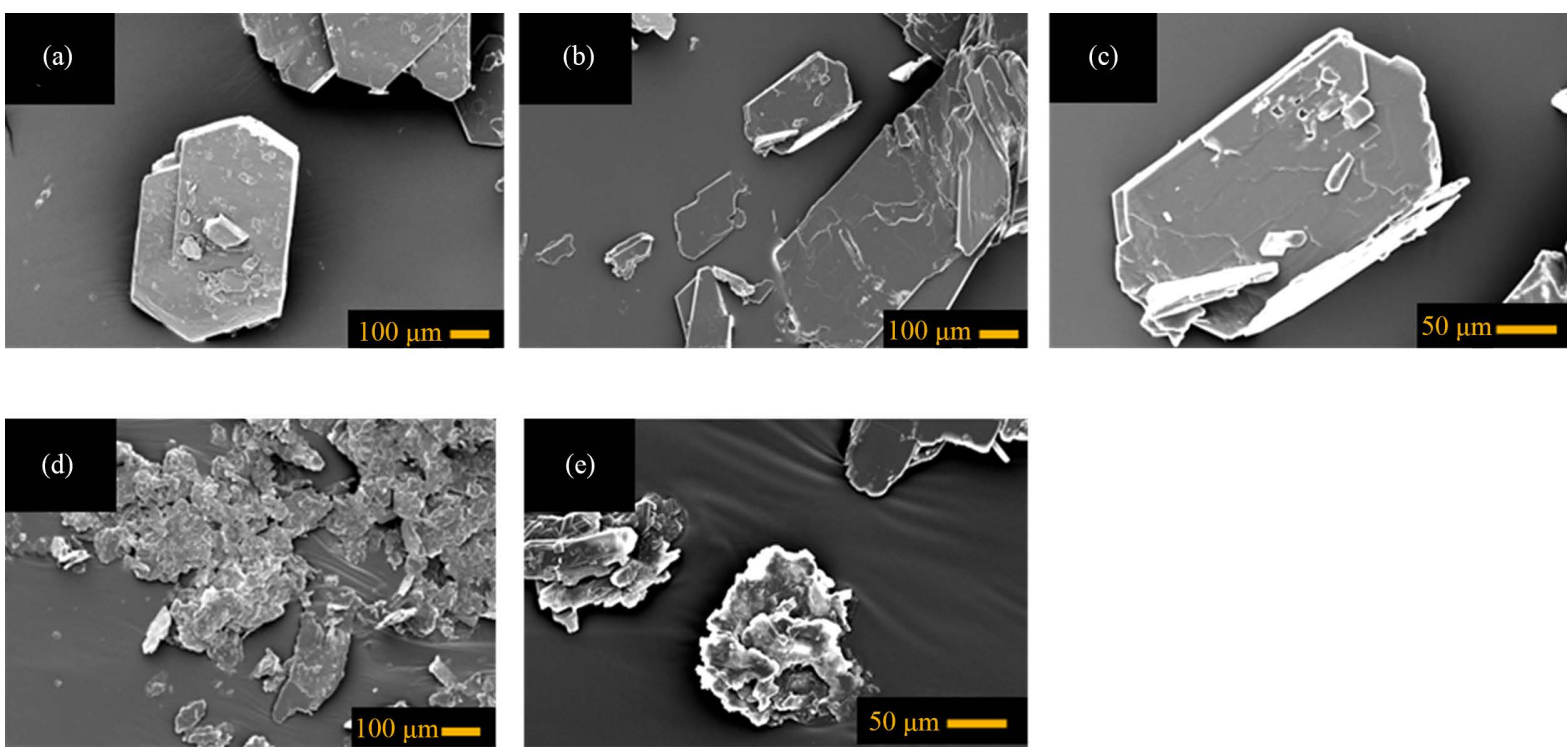

Figure 4. SEM micrographs of crystals: (a) L-Val single crystal (100×), (b) D-Leu/L-Val = 0.03/99.97 (100×), (c) D-Leu/ $\mathrm{L}-\mathrm{Val}=0.03 / 99.97(300 \times)$, (d) L-Leu/L-Val = 2/98 $(100 \times)$, and $(\mathrm{e}) \mathrm{L}-\mathrm{Leu} / \mathrm{L}-\mathrm{Val}=2 / 98(400 \times)$, where the molar ratio between L-Val and L-Leu or D-Leu in the crystals is represented as L-Leu/L-Val [mol/mol] and the magnification is denoted in parentheses.
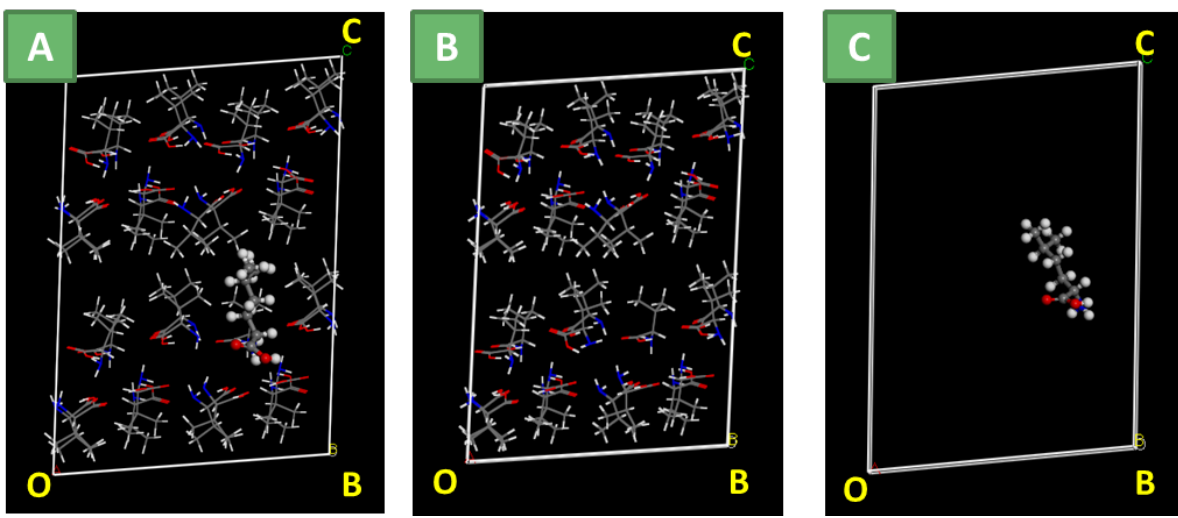

Figure 5. Crystal structure (projection figures from the $a$-axis). This figure shows the case of D-Leu in an L-Val crystal lattice. L-Val molecules are shown as stick models, D-Leu molecules are shown as ball-and-stick models, and the void represents vacuum. Structure A: L-Val $4 \times 4 \times 2$ crystal lattice in which one of the L-Val molecules in the (100) surface was substituted with D-Leu and the lattice was optimized geometrically using Materials Studio. Structure B: Substituted D-Leu was removed from structure A. Structure C: Only D-Leu remained in structure A.

Table 1. Calculated interaction energies between guest amino acid molecules and an L-Val crystal $[\mathrm{kJ} / \mathrm{mol}]$.

\begin{tabular}{ccccc}
\hline Guest amino acid & $E_{\mathrm{A}}$ & $E_{\mathrm{B}}$ & $E_{\mathrm{C}}$ & $E_{\mathrm{I}}$ \\
\hline L-Val & 123 & 128 & 29 & -34 \\
L-Leu & 120 & 136 & 15 & -31 \\
D-Leu & 340 & 143 & 12 & 185 \\
\hline
\end{tabular}

"Guest amino acid" means substituted amino acid. Materials Studio was used to estimate the interaction energy (module: forcite, potential function: cvff). 


$$
E_{\mathrm{I}}=E_{\mathrm{A}}-\left(E_{\mathrm{B}}+E_{\mathrm{C}}\right)
$$

where $E_{\mathrm{A}}, E_{\mathrm{B}}$, and $E_{\mathrm{C}}$ were calculated as the total energy of structure $\mathrm{A}$, structure $\mathrm{B}$, and structure C, respectively.

The estimated interaction energy is shown in Table 1. In the case where L-Val was used as a guest amino acid, $E_{\mathrm{I}}$ represents the interaction energy between a molecule of L-Val and an L-Val crystal. A comparison of the interaction energies reveals that the energy of D-Leu was remarkably higher than those of L-Leu and L-Val. The interaction energy is correlated with the likelihood of incorporation. In the case of a high interaction energy, the crystal lattice became unstable and difficult to construct; therefore, D-Leu was difficult to be incorporated.

\section{Conclusion}

A mixture of L-Leu and L-Val crystals was formed as a solid solution randomly constructed by L-Leu and L-Val in the crystal lattice. By contrast, D-Leu was not incorporated into L-Val crystals and did not result in the formation of a solid solution when $x_{\text {D-Leu }}$ was less than 0.12 in the mother liquor because the interaction energy between a D-Leu molecule and the L-Val crystal lattice was remarkably higher than that between an L-Leu molecule and the L-Val crystal lattice.

\section{References}

[1] Kim, H.K., Suzuki, T., Saito, K., Yoshida, H., Kobayashi, H., Kato, H. and Katayama, M. (2012) Effects of Exercise and Amino Acid Supplementation on Body Composition and Physical Function in Community-Dwelling Elderly Japanese Sarcopenic Women: A Randomized Controlled Trial. Journal of the American Geriatrics Society, 60, 16-23. http://dx.doi.org/10.1111/j.1532-5415.2011.03776.x

[2] Katsanos, C.S., Kobayashi, H., Sheffield-Moore, M., Aarsland, A. and Wolfe, R.R. (2006) A High Proportion of Leucine is Required for Optimal Stimulation of the Rate Of Muscle Protein Synthesis by Essential Amino Acids in the Elderly. American Journal of Physiology_Endocrinology and Metabolism, 291, E381-E387. http://dx.doi.org/10.1152/ajpendo.00488.2005

[3] Kamei, T., Hasegawa, K., Yokota, M., Doki, N. and Shimizu, K. (2009) AFM Observation of the Blocking of Step Motion Caused by L-Norleucine on (100) and (001) Faces of an L-Isoleucine Crystal. Journal of Chemical Engineering of Japan, 42, 464-470. http://dx.doi.org/10.1252/jcej.08we311

[4] Harding, M.M. and Howieson, R.M. (1976) L-Leucine. Acta Crystallographica Section B, 32, 633-634. http://dx.doi.org/10.1107/S0567740876012405

[5] Torii, K. and Iitaka, Y. (1970) The Crystal Structure of L-Valine. Acta Crystallographica Section B, 26, $1317-1326$. http://dx.doi.org/10.1107/S0567740870004065

[6] Torii, K. and Iitaka, Y. (1971) The Crystal Structure of L-Isolucine. Acta Crystallographica Section B, 27, $2237-2246$. http://dx.doi.org/10.1107/S0567740871005612

[7] Kamei, T., Hasegawa, K., Kashiwagi, T., Suzuki, E., Yokota, M., Doki, N. and Shimizu, K. (2008) Mechanism of Mutual Incorporationof L-Isoleucine and Isomorphic Amino Acids in Batch Crystallization. Organic Process Research \& Development, 12, 850-854. http://dx.doi.org/10.1021/op800093x

[8] Kamei, T., Hasegawa, K., Fuke, I., Nagai, H., Yokota, M., Doki, N. and Shimizu, K. (2008) Mechanism of Mutual Incorporation of Branched Chain Amino Acids and Isomorphic Amino Acids in Batch Crystallization. Journal of Chemical Engineering of Japan, 41, 460-469. http://dx.doi.org/10.1252/jcej.07we284

[9] Dalhus, B. and Görbitz, C.H., (1999) Molecular Aggregation in Selected Crystalline 1:1 Complexes of Hydrophobic D- and L-Amino Acids. I. The L-Isoleucine Series. Acta Crystallographica Section B, 55, 424-431. http://dx.doi.org/10.1107/S0108768198013494

[10] Dalhus, B. and Görbitz, C.H. (1999) Molecular Aggregation in Selected Crystalline 1:1 Complexes Of Hydrophobic Dand L-Amino Acids. II. The D-Norleucine Series. Acta Crystallographica Section C, 55, 1105-1112. http://dx.doi.org/10.1107/S0108270199003649

[11] Dalhus, B. and Görbitz, C.H. (1999) Molecular Aggregation in Selected Crystalline 1:1 Complexes of Hydrophobic Dand L-Amino Acids. III. The L-Leucine and L-Valine Series. Acta Crystallographica Section C, 55, 1547-1555. http://dx.doi.org/10.1107/S0108270199006162

[12] Dauber-Osguthorpe, P., Roberts, V.A., Osguthorpe, D.J., Wolff, J., Genest, M. and Hagler, A.T. (1988) Structure and Energetics of Ligand Binding to Proteins: Escherichia coli Dihydrofolate Reductase-Trimethoprim, a Drug-Receptor System. Proteins: Structure, Function, and Bioinformatics, 4, 31-47. http://dx.doi.org/10.1002/prot.340040106 
[13] Anuar, N., Daud, W.R.W., Roberts, K.J., Kamarudin, S.T. and Tasirin, S.M. (2012) Morphology and Associated Survface Chemistry of L-Isoleucine Crystals Modeled under the Influence of L-Leucine Additive. Crystal Growth \& Design, 12, 2195-2203. http://dx.doi.org/10.1021/cg200266e

\section{Submit or recommend next manuscript to SCIRP and we will provide best service for you:}

Accepting pre-submission inquiries through Email, Facebook, Linkedin, Twitter, etc A wide selection of journals (inclusive of 9 subjects, more than 200 journals)

Providing a 24-hour high-quality service

User-friendly online submission system

Fair and swift peer-review system

Efficient typesetting and proofreading procedure

Display of the result of downloads and visits, as well as the number of cited articles

Maximum dissemination of your research work

Submit your manuscript at: http://papersubmission.scirp.org/ 